$\begin{array}{llllllllllll}A & R & T & I & C & U & L & O\end{array}$

\title{
PROSPECTIVA INDUSTRIAL DE LAS Minicadenas Productivas Hortifruticolas DE Bolívar*
}

\author{
Por: Julio Amézquita López \\ Erik Baldovino Villacob
}

\section{RESUMEN}

El presente artículo analiza desde el enfoque de la prospectiva el desarrollo industrial de las minicadenas hortifrutícolas para plantear opciones estratégicas tendientes a mejorar el desarrollo agroindustrial del Departamento de Bolivar, y hacer de las minicadenas hortifrutícolas un cluster económicamente competitivo y socialmente sostenible. Para ello se hace uso de técnicas como el análisis estructural (método $M I C M A C)$ y juego de actores (método MACTOR), como una forma de promover una reflexión estratégica entre los actores de la cadena. Como resultados del proceso se encuentra que son necesarias acciones principalmente a nivel de productos innovadores, biotecnología, Impacto ambiental, tecnologías de procesamiento, alianzas estratégicas, disposición a la asociatividad, acuerdos comerciales internacionales, productividad, vocación exportadora y márgenes de ingreso, como fuentes para mejorar el desarrollo del sector.

\section{La Prospectiva como Herramienta de Planificación}

* El presente artículo hace parte del Proyecto de Maestría del Ingeniero Julio Amezquita, titulado: Prospectiva $\quad \mathrm{y}$ Competitividad de las $\mathrm{C}$ a $\mathrm{d}$ e $\mathrm{n}$ a $\mathrm{s}$ Hortifrutícolas en el Departamento de Bolívar. os retos competitivos de la economía globalizada exigen cada día más una visión estratégica de los territorios, las empresas, las instituciones y los productos en busca de seguir un camino coherente de largo plazo hacia un desarrollo social, económico y ambiental. Para conseguir tal fin, la prospectiva se ha convertido en una valiosa herramienta para buscar los mejores caminos que guíen a la humanidad hacia un mejor futuro, partiendo de las posibles visiones del futuro y desarrollando acciones en el presente que nos lleven hacia un futuro deseado.

En este proceso de búsqueda de los caminos hacia el futuro, la prospectiva toma una visión sistémica de los problemas e involucra a todos los actores que intervienen dentro de dicho sistema. Además, hace uso de tres valiosos elementos para lograr el éxito del proceso. El primero de ellos es la reflexión colectiva en la cual es importante lograr consensos entre los actores del sistema sobre todo para llegar a una visión conjunta del futuro y una apuesta del sistema para 
lograr un escenario. Así también, la apropiación como proceso transversal de la prospectiva, tiene una gran importancia dado que a partir de ella los actores tendrán una mayor o menor apropiación sobre las apuestas y visiones de futuro. En tercer lugar, la acción estratégica es el camino para hacer realidad la visión de futuro hacia la cual se le apuesta desde la reflexión estratégica. De allí, es que Michel Godet (2000) hace énfasis en que "La anticipación no tiene mayor sentido si no es que sirve para esclarecer la acción". "Esa es la razón por la cual la prospectiva y la estrategia son generalmente indisociables. De ahí viene la expresión de prospectiva estratégica. Sin embargo, la complejidad de los problemas y la necesidad de plantearlos colectivamente imponen el recurso a métodos que sean tan rigurosos y participativos como sea posible, al objeto de que las soluciones sean reconocidas y aceptadas por todos" ${ }^{1}$

\section{El Problema del Desarrollo Agroindustrial de las Minicadenas Hortifrutícolas de Bolívar}

El proyecto desarrollado se centro en el proceso de reflexión colectiva con actores de las minicadenas hortifrutícolas del departamento de Bolívar con el fin de realizar un aporte a varios procesos de planificación realizados a nivel nacional y regional tales como la agenda interna de productividad y competitividad, y la agenda departamental de ciencia, tecnología e innovación. En los talleres realizados se contó con la participación de investigadores, empresarios, representantes del estado y asociaciones de productores. El proceso de reflexión se dio a partir de tres interrogantes que definen la problemática en estudio:

¿Qué puede pasar con la competitividad de estas cadenas agroindustriales en un futuro (de 2005 al 2015), por ejemplo? En el departamento de Bolívar no hemos comprendido cabalmente los obstáculos a la competitividad que se reflejan como cuellos de botella de las cadenas hortifrutícolas y buscar entonces soluciones consistentes con nuestras capacidades que nos permitan: Tener unas mejores tasas de inversión y creación de nuevas empresas, mayor productividad y empleo, así como un desarrollo tecnológico regional y de fomento a la producción y la compra nacional de bienes de capital dirigidos al sector de alimentos y bebidas. ¿Qué podemos hacer para mejorar el desempeño competitivo y social de las cadenas Hortifrutícolas en Bolívar?: Algunos empresarios y el gobierno (Agenda Interna de Productividad y Competitividad, Agendas de Ciencia y Tecnología) se han interesado por generar políticas tendientes a mejorar la capacidad competitiva de la economía colombiana, principalmente en los mercados internacionales a través de Acuerdos de Competitividad y Eslabonamientos Productivos, mientras que algunos actores están más preocupados por mejorar

${ }^{1}$ Godet, Michel. La caja de herramientas de la prospectiva estratégica. Cuadernos de LIPS No. 5., Laboratoire d'Investigation Prospective et Stratégique. Abril de 2000. P. 2. 
los índices de la calidad de vida de nuestros compatriotas y focalizarse en la erradicación de la pobreza.

En el marco de una economía mundial globalizada la autosuficiencia alimentaria y la competitividad de las empresas parecen divergir cada día más respecto a sus intereses, y sin embargo ambas necesidades resultan ser factores primordiales para mejorar el bienestar de la población local o regional y el desarrollo industrial sostenible. ¿Para quién debe trabajar la cadena productiva a futuro?, ¿Cómo podemos construir escenarios donde cambiemos el paradigma del control alimentario globalizado y ejercido por otros?

¿Cuales acciones, programas o proyectos conjuntos pueden mejorar la productividad agrícola e industrial, o la competitividad internacional, o a la innovación empresarial con valor agregado, empleo y conocimiento?: El problema es de diseño estratégico regional con miras a los acuerdos de competitividad y en un horizonte de tiempo que abarca hasta el 2015: La industria en cuestión no aporta una interrelación más dinámica de la gran empresa con las PYMES, igual fenómeno sucede con el sector de producción primaria y el de transformación dentro de los cuales existe poca interrelación y encadenamiento, que permita impulsar un proceso

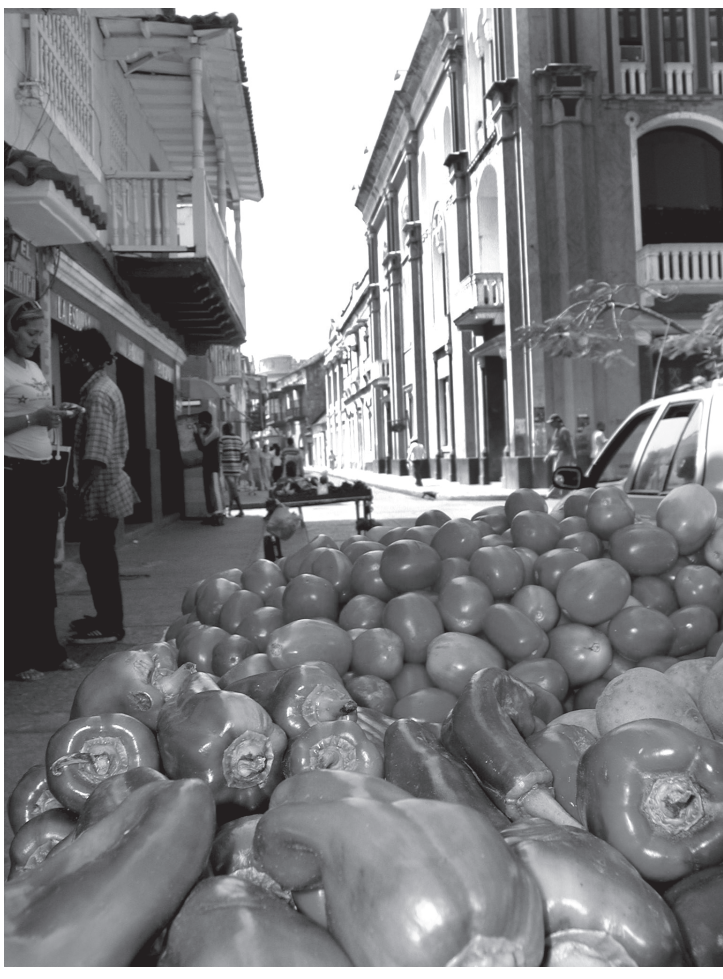

Foto: sinérgico en la generación de ventajas competitivas para las empresas del subsector, en el sentido, de que la gran empresa no jalona el desarrollo de las PYMES, y no se provoca un proceso de especialización dentro de la cadena productiva.

Este problema de Competitividad debe estar matizado por el de seguridad alimentaria, ya que los estudios relacionados con el potencial exportador recogen sectorialmente las expectativas pero no relacionan la oferta exportadora de frutas y hortalizas con la demanda interna de procesamiento de alimentos, bebidas y otros químicos y a su vez con el aumento de la capacidad en este segundo nivel.

\section{La Visión del Sistema como Cluster}

"Se entiende comúnmente por complejo productivo o cluster una concentración sectorial y/o geográfica de empresas que se desempeñan en las mismas actividades 
o en actividades estrechamente relacionadas —tanto hacia atrás, hacia los proveedores de insumos y equipos, como hacia adelante y hacia los lados, hacia industrias procesadoras y usuarias así como a servicios y actividades estrechamente relacionadas - con importantes y cumulativas economías externas, de aglomeración y especialización (por la presencia de productores, proveedores y mano de obra especializada y de servicios anexos específicos al sector) y con la posibilidad de llevar a cabo una acción conjunta en búsqueda de eficiencia colectiva". ${ }^{2}$ Para realizar un análisis sistémico de la situación para las minicadenas hortofrutícolas del departamento, no sólo basta con ver la cadena sólo como los procesos de cosecha - transformación industrial Comercialización, sino que además es necesario ver los agentes conexos a este proceso, en donde se encuentran el estado, las instituciones para la colaboración y el medio académico e investigativo. Una aproximación al sistema basado en los procesos y productos de las cadenas productivas y sus agentes conexos se puede apreciar en la siguiente figura.

Figura 1

Estructura Simplificada de la Cadena Hortifrutícola

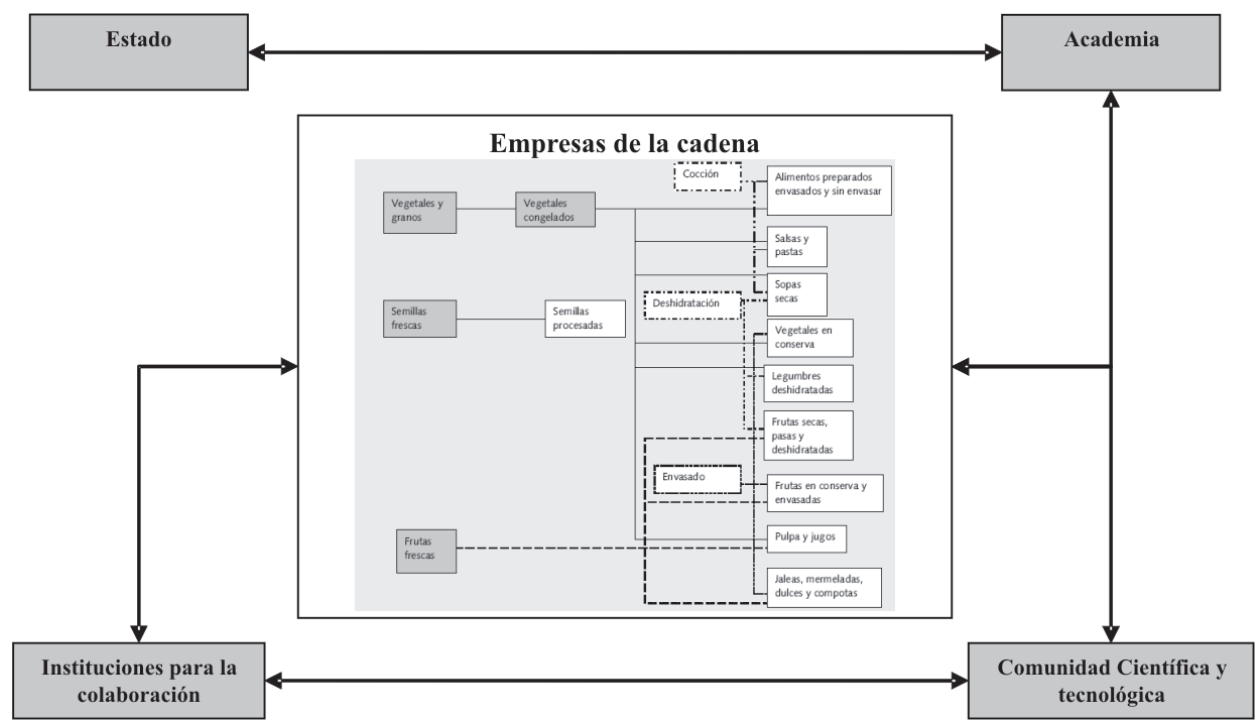

Fuente: DNP - Análisis de la Cadena Hortifrutícola. Diseño de los Autores

Este esquema general puede aplicarse a cada cultivo en Bolívar. Pero se requiere entender el proceso de encadenamiento conforme a los eslabones logísticos que lo conforman: Productores o cultivadores en sector agrícola, operadores de poscosecha, transportistas, proveedores de empaque y embalaje, almacenadoras y centros de acopio, distribuidores comercializadores, transformadores industriales (alimentos, bebidas, químicos), exportadores e importadores de frutas o verduras y fondos de inversión.

2 Ramos, Joseph. Complejos productivos en torno a los recursos naturales: ¿una e s t r a t e g i a prometedora? Artículo publicado en: Martine Dirven (compiladora). Apertura económica y (des)encadenamientos p r o d u c t i vo s. Reflexiones sobre el complejo lácteo en América Latina. CEPAL. Julio de 2001. P. 33. 


\section{Factores Claves para el Desarrollo de las Minicadenas Hortifrutícolas de Bolívar}

Para realizar el análisis prospectivo de los factores claves para el desarrollo agroindustrial de las minicadenas hortofrutícolas de Bolívar se hizo uso de la herramienta del Análisis Estructural. Para ello se partió de la clasificación de las 31 variables importantes en la actualidad y a futuro para la evolución del sistema analizado, y previa validación con los actores participantes en el taller prospectivo se procedió a realizar el análisis de impactos cruzados por parte un grupo de actores y expertos por medio de una matriz de impactos cruzados. Los resultados de las influencias y dependencias atribuidas a las variables se muestran en el siguiente gráfico:

Grafico 1

Mapa de Influencias y Dependencias Directas

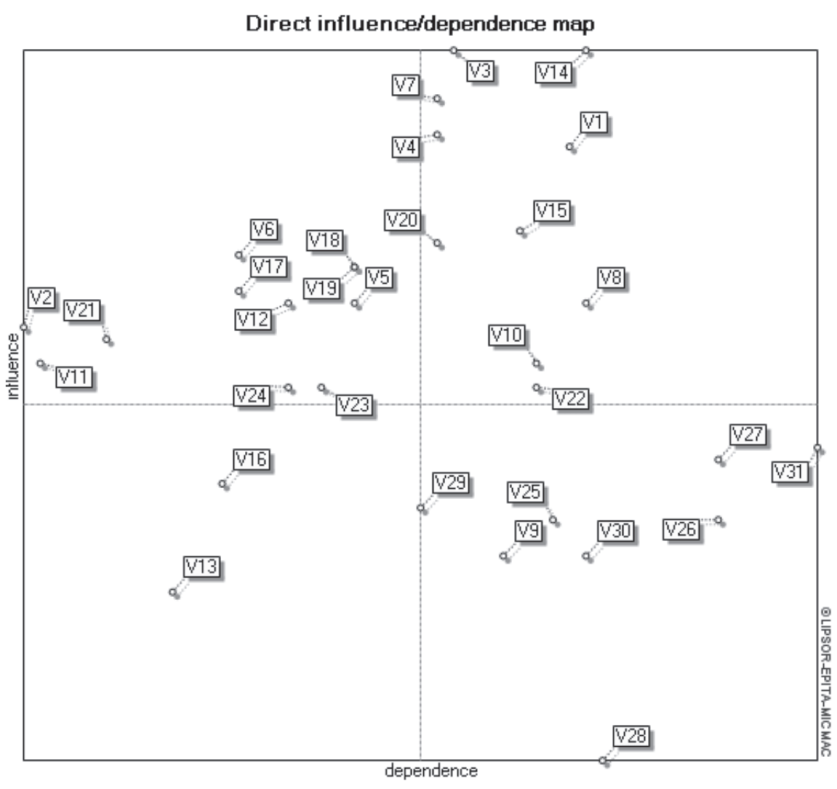

Fuente: Datos de Salida Software MICMAC con Base en Opiniones de los Actores

clasificación se parte de la matriz de impactos directos, elevándola a la "n" (enésima) potencia, donde "n" es la potencia en la cual no existe ningún cambio significativo en las relaciones de influencia / dependencia de las variables. A este nivel se dice que se alcanza la estabilidad del sistema. Para el presente caso se realizaron cuatro iteraciones de la matriz de impactos directos.
Sin embargo, se hizo uso de las relaciones indirectas entre las variables para confirmar la importancia de ciertas variables, pero de igual manera para develar ciertas variables que en razón de sus acciones indirectas juegan un papel principal (y que la clasificación directa no pone de manifiesto). ${ }^{3}$ En otros términos, esta forma de analizar las relaciones entre las variables parte del hecho de que una de ellas puede influir sobre otra(s) por intermedio de una tercera variable, lo cual pone de manifiesto una mayor importancia de algunas variables. Los cambios en las posiciones de las variables o bien en su capacidad de explicar el sistema se pueden evidenciar en el siguiente cuadro. 


\section{Cuadro 1}

Cambios en las Relaciones de Influencia

y Dependencia de las Variables

\begin{tabular}{|c|c|c|c|c|c|}
\hline \multicolumn{3}{|c|}{ Clasificación por influencias } & \multicolumn{3}{|c|}{ Clasificación por dependencias } \\
\hline \multicolumn{2}{|c|}{ MID } & \multirow{2}{*}{$\begin{array}{c}\text { MII } \\
\text { Variable }\end{array}$} & \multicolumn{2}{|c|}{ MID } & \multirow{2}{*}{$\begin{array}{r}\text { MII } \\
\text { Variable }\end{array}$} \\
\hline Rank & Variable & & Rank & Variable & \\
\hline 1 & $3-v_{3}$ & $3-\sqrt{3}$ & 1 & $31-\mathrm{V} 31$ & $31-V_{31}$ \\
\hline 2 & $14-\mathrm{V} 14$ & \begin{tabular}{|c|}
$14-\mathrm{V} 14$ \\
\end{tabular} & 2 & & $27-\mathrm{V} 27$ \\
\hline 3 & $7-\sqrt{ } 7$ & $7-v 7$ & 3 & $27-\sqrt{2} 27$ & $26-\mathrm{V} 26$ \\
\hline 4 & $4-\mathrm{V}_{4}$ & $1-\mathrm{V}_{1}$ & 4 & $28-\mathrm{V} 28$ & \begin{tabular}{|l|}
$28-\mathrm{V} 28$ \\
\end{tabular} \\
\hline$\frac{4}{5}$ & 1-V1 & $4-\mathrm{V}_{4}$ & 5 & $8-\mathrm{v}^{2}$ & $8-\mathrm{vg}$ \\
\hline 6 & $15-\mathrm{V} 15$ & \begin{tabular}{|l}
$15-\mathrm{V} 15$ \\
\end{tabular} & 6 & $14-\mathrm{V} 14$ & $30-\mathrm{v} 30$ \\
\hline 7 & $20-\mathrm{V} 20 \mathrm{C}$ & 18 -V18 & 7 & $30-\mathrm{V} 30$ & $14-\mathrm{V} 14$ \\
\hline 8 & $6-v_{6}$ & $6-V_{6}$ & 8 & $1-\mathrm{V}_{1}$ & $1-\mathrm{V}_{1}$ \\
\hline 9 & $18-\mathrm{V} 18 \mathrm{r}$ & $20-\mathrm{V} 20$ & 9 & $25-\mathrm{V}_{2} 5$ & $25-\mathrm{V} 25$ \\
\hline 10 & $19-v_{11}$ & \begin{tabular}{|c|}
$19-\mathrm{V} 19$ \\
\end{tabular} & 10 & $10-\mathrm{V} 10^{0}$ & $15-\mathrm{V} 15$ \\
\hline 11 & $17-\mathrm{V} 17$ & \begin{tabular}{|c|}
$17-\mathrm{V} 17$ \\
\end{tabular} & 11 & $22-\mathrm{V} 22^{\circ}$ & $9-v_{9}$ \\
\hline 12 & $5 . \mathrm{v5}$ & $12-\mathrm{V} 12$ & 12 & $15-\mathrm{V} 15$ & $10-\mathrm{V} 10$ \\
\hline 13 & $8 \cdot \mathrm{v} 8$ & $8-\mathrm{v} 8$ & 13 & $9-v_{9}$ & $22-\mathrm{V} 22$ \\
\hline 14 & $12-\mathrm{V}_{12}$ & $5-v_{5}$ & 14 & $3-\mathrm{V} 3$ & $3-\sqrt{3}$ \\
\hline 15 & $2 \cdot \mathrm{V}_{2}$ & $2 \cdot \mathrm{V}_{2}$ & 15 & $4-\mathrm{v}_{4} \mathrm{O}$ & $7-\mathrm{V7}$ \\
\hline 16 & $21-\mathrm{v} 21$ & \begin{tabular}{|l|}
$21-\mathrm{V} 21$ \\
\end{tabular} & 16 & $7-v 7$ & $4-V_{4}$ \\
\hline 17 & $10-\mathrm{V} 10 \mathrm{O}$ & $11-\mathrm{V} 11$ & 17 & $20-\mathrm{V}_{20}$ & $20-\mathrm{V} 20$ \\
\hline 18 & $11-\mathrm{V} 11$ & $10-\mathrm{V} 10$ & 18 & $29-\mathrm{V}_{2} \mathrm{a}$ & $29-\mathrm{V} 29$ \\
\hline 19 & $22 \cdot \mathrm{V} 22$ & $24-\mathrm{V} 24$ & 19 & $5-\mathrm{V} 5$ & $5-\mathrm{v} 5$ \\
\hline 20 & $23-\mathrm{V} 23^{3}$ & $22-\mathrm{v} 22$ & 20 & $18-\mathrm{V} 18$ & $19-\mathrm{V} 19$ \\
\hline 21 & $24-\mathrm{V} 24$ & $23-\mathrm{V} 23$ & 21 & $19-\mathrm{v} 19$ & $18-\mathrm{V} 18$ \\
\hline 22 & $31-\sqrt{31}$ & \begin{tabular}{|l|}
$31-\mathrm{V} 31$ \\
\end{tabular} & 22 & $23-\mathrm{V}_{2} 3$ & \begin{tabular}{|l|}
$23-\mathrm{V} 23$ \\
\end{tabular} \\
\hline 23 & $27-\sqrt{2} 27$ & \begin{tabular}{|l|}
$27-\mathrm{V} 27$ \\
\end{tabular} & 23 & $12-\mathrm{V}_{12}$ & $17-v 17$ \\
\hline 24 & $16-\mathrm{v} 16$ & \begin{tabular}{|l|}
$16-\mathrm{V} 16$ \\
\end{tabular} & 24 & $24-\mathrm{V} 24$ & $12-\mathrm{V} 12$ \\
\hline 25 & $29-\mathrm{V} 29$ & \begin{tabular}{|l|}
$29-\mathrm{V} 29$ \\
\end{tabular} & 25 & $6-\mathrm{V}^{6}$ & $24-\mathrm{V} 24$ \\
\hline 26 & $25-\mathrm{V} 25$ & $9-v_{0}$ & 26 & $17-\mathrm{v} 17$ & $6-\mathrm{v}^{\circ}$ \\
\hline 27 & $26-\mathrm{V} 20 \mathrm{O}$ & $30-\mathrm{V} 30$ & 27 & $16-\mathrm{V} 16$ & $16-\mathrm{V} 16$ \\
\hline 28 & $9 . \mathrm{vg}$ & 26 - V 26 & 28 & $13-\mathrm{V} 13$ & $13-\mathrm{V} 13$ \\
\hline 29 & $30-\mathrm{V}_{3} \mathrm{Or}$ & 25 - V25 & 29 & $21-\mathrm{V} 21$ & $21-\mathrm{V} 211$ \\
\hline 30 & $13-\mathrm{V} 13$ & 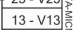 & 30 & $11-\mathrm{V} 110$ & $2-\mathrm{v} 2$ \\
\hline \begin{tabular}{|l}
31 \\
\end{tabular} & $28-v 28$ & \begin{tabular}{|l|l}
$28-\mathrm{V} 28$ \\
\end{tabular} & 31 & $2-\mathrm{V}^{2}$ & - \\
\hline
\end{tabular}

Fuente: Datos de Salida Software MICMAC con Base en Opiniones de los Actores

El gráfico 2 permite visualizar las posiciones relativas de las variables a partir de sus relaciones indirectas (matriz de impactos indirectos). De acuerdo con éste gráfico se pueden apreciar las clasificaciones de las variables o bien su importancia para la evolución del sistema.

\section{Grafico 2}

Mapa de Influencias y Dependencias Indirectas

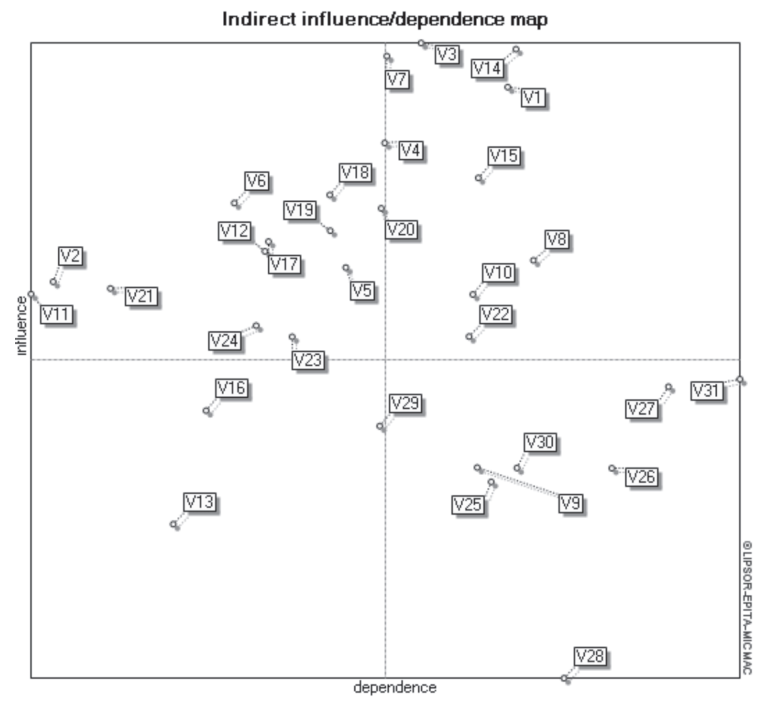

Fuente: Datos de Salida Software MICMAC con Base en Opiniones de los Actores 
De esta forma, se pueden identificar las variables claves que explican la evolución del sistema, entendidas como aquellas con mayor motricidad, y las variables más dependientes. Estas variables tienen la capacidad de explicar el sistema en estudio. Partiendo del criterio de Pareto, las divisiones del gráfico dan lugar a las posiciones que asumen las variables en el comportamiento del sistema estudiado. Así, encontraremos, variables de entrada, de enlace, de salida, y las que definitivamente no son importantes para el sistema.

Si tenemos en cuenta sólo el 5\% de las relaciones de influencia, el gráfico 3 nos muestra las relaciones entre las variables más importantes del sistema.

\section{Gráfico 3}

\section{Influencias Indirectas entre Variables}

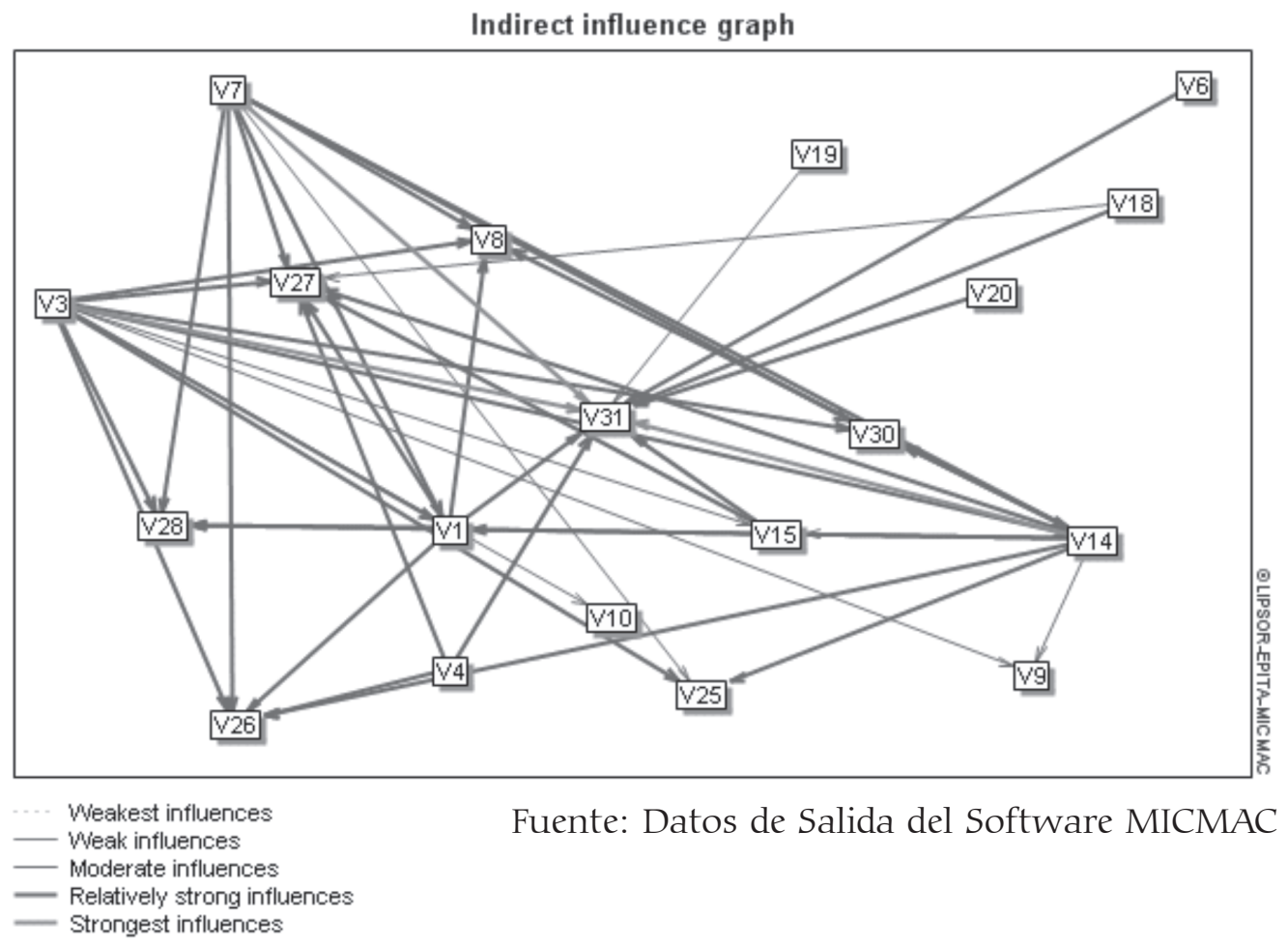

De esta forma, las variables más importantes para el comportamiento actual y futuro de competitividad y el desarrollo industrial de la cadena hortifrutícola de Bolívar son las que aparecen en el cuadro 2.

Así, la influencia que se pueda ejercer sobre estas variables explica la evolución del sistema en estudio. En este sentido, se deben plantear objetivos a futuro sobre las variables más influyentes de tal forma que se pueda ejercer un impacto significativo para el logro de los objetivos. 
Cuadro 2

Variables Claves del Sistema

\begin{tabular}{|c|c|c|}
\hline Tipo de Variable & Variable & Código \\
\hline \multirow{7}{*}{$\begin{array}{l}\text { Variables de } \\
\quad \text { entrada }\end{array}$} & Innovación y oferta de más productos con valor agregado & V3 \\
\hline & $\begin{array}{l}\text { Aplicación de biotecnología (Semillas mejoradas, métodos } \\
\text { naturales para riesgos biológicos, etc.) }\end{array}$ & V4 \\
\hline & $\begin{array}{l}\text { Manejo del impacto ambiental (implementación de normas } \\
\text { técnicas de manejo ambiental) }\end{array}$ & V6 \\
\hline & $\begin{array}{l}\text { Inversiones en tecnologías de procesamiento (maquinarias, } \\
\text { equipos, laboratorios, TIC's, Automatización, etc.) }\end{array}$ & V7 \\
\hline & $\begin{array}{l}\text { Realización de Alianzas estratégicas con empresas afi nes del } \\
\text { sector }\end{array}$ & V18 \\
\hline & $\begin{array}{l}\text { Disposición a la asociatividad y Desarrollo de } \\
\text { encadenamientos productivos }\end{array}$ & V19 \\
\hline & $\begin{array}{l}\text { Acuerdos comerciales internacionales (TLC, CAN, } \\
\text { MERCOSUR, etc.) }\end{array}$ & V20 \\
\hline \multirow{3}{*}{$\begin{array}{l}\text { Variables de } \\
\quad \text { enlace }\end{array}$} & $\begin{array}{l}\text { Exigencias en aumento de la productividad (agrícola e } \\
\text { industrial) y optimización de uso de suelos }\end{array}$ & V1 \\
\hline & $\begin{array}{l}\text { Vocación exportadora de la empresa e internacionalización de } \\
\text { la empresa }\end{array}$ & V14 \\
\hline & Mejorar márgenes de ingresos desde el cultivo & V15 \\
\hline \multirow{9}{*}{$\begin{array}{l}\text { Variables } \\
\text { resultado }\end{array}$} & $\begin{array}{l}\text { Mejoras en logística de abastecimiento, almacenamiento y } \\
\text { distribución (empaque, transporte, embalaje etc.) }\end{array}$ & V8 \\
\hline & Generación de empleo semicalificado y calificado en la región & V9 \\
\hline & $\begin{array}{l}\text { Capacitación, entrenamiento y certificación de competencias } \\
\text { laborales del personal }\end{array}$ & V10 \\
\hline & $\begin{array}{l}\text { Disponibilidad de mejores canales de comercialización y } \\
\text { autonomía frente a Comercializadoras Internas (Brokers) }\end{array}$ & V25 \\
\hline & $\begin{array}{l}\text { Relación con Cdt's, universidades, Laboratorios, Centros de } \\
\text { investigación o innovación para el desarrollo de I\&D }\end{array}$ & V26 \\
\hline & $\begin{array}{l}\text { Disponibilidad de financiamiento público o privado (créditos, } \\
\text { cofinanciación) para proyectos }\end{array}$ & V27 \\
\hline & $\begin{array}{l}\text { Utilización de estímulos tributarios para el desarrollo de } \\
\text { proyectos de innovación }\end{array}$ & V28 \\
\hline & $\begin{array}{l}\text { Confiabilidad en los marcos legales respecto a la protección de } \\
\text { la libre competencia }\end{array}$ & V30 \\
\hline & Promoción de producción con uso alternativos y mercadeo & V31 \\
\hline
\end{tabular}

Fuente: Elaboración Propia con Base en Datos de Salida del Software MICMAC

\section{Los Objetivos Estratégicos y las Posiciones de los Actores}

Priorizados los factores claves para el desarrollo agroindustrial de las minicadenas hortifrutícolas de bolívar se evidencia que los objetivos hacia los cuales le deben aportar los actores de la cadena se relacionan con los listados indicados en el Cuadro 3.

En este sentido, la planificación del desarrollo del sector requiere de altas inversiones para su modernización y para poder alcanzar una posición competitiva y sostenible tanto para los agricultores como para lo industriales.

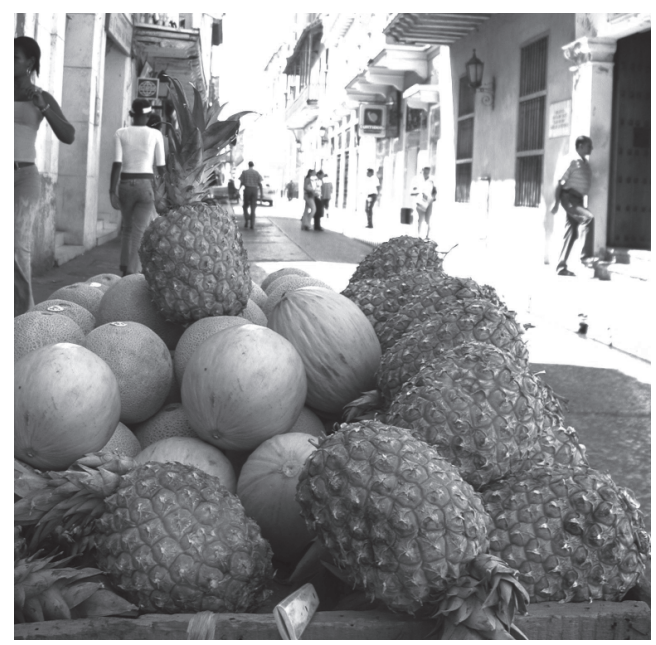


Cuadro 3

Objetivos Estratégicos

\begin{tabular}{|l|l|l|}
\hline $\mathbf{N}^{\circ}$ & OBJETIVO & DESCRIPCION \\
\hline 1 & Productos innovadores & $\begin{array}{l}\text { Desarrollo de una oferta de productos innovadores con alto valor } \\
\text { agregado y usos alternativos }\end{array}$ \\
\hline 2 & Biotecnología & $\begin{array}{l}\text { Implementar programas para aumentar la aplicación de las } \\
\text { biotecnologías en los procesos de la cadena, en conjunto con CDT's, } \\
\text { centros de innovación etc. }\end{array}$ \\
\hline 3 & Impacto ambiental & $\begin{array}{l}\text { Implementación de normas técnicas para el manejo del impacto } \\
\text { ambiental }\end{array}$ \\
\hline 4 & $\begin{array}{l}\text { Tecnologías } \\
\text { procesamiento }\end{array}$ & $\begin{array}{l}\text { Desarrollar proyectos o invertir en el mejoramiento de las tecnologías de } \\
\text { procesamiento de la agroindustria relacionada con la cadena }\end{array}$ \\
\hline 5 & Alianzas estratégicas & $\begin{array}{l}\text { Realización de alianzas estratégicas con empresas afines del sector para } \\
\text { afrontar los retos competitivos }\end{array}$ \\
\hline 6 & $\begin{array}{l}\text { Disposición a la } \\
\text { asociatividad }\end{array}$ & $\begin{array}{l}\text { Sensibilización y disponibilidad de las directivas hacia la asociatividad y } \\
\text { el desarrollo de encadenamientos productivos }\end{array}$ \\
\hline 7 & $\begin{array}{l}\text { Acuerdos comerciales } \\
\text { internacionales }\end{array}$ & $\begin{array}{l}\text { Asumir posiciones preactivas frente a los acuerdos comerciales vigentes } \\
\text { y por desarrollarse }\end{array}$ \\
\hline 8 & Productividad & $\begin{array}{l}\text { Desarrollar e invertir en proyectos que mejoren la productividad ind ustrial } \\
\text { y agrícola de la cadena hortofrutícola }\end{array}$ \\
\hline 9 & Vocación exportadora & $\begin{array}{l}\text { Mejorar la vocación exportadora e internacionalización de las empresas } \\
\text { relacionadas con la cadena }\end{array}$ \\
\hline 10 & Márgenes de ingreso & $\begin{array}{l}\text { Desarrollar proyectos y/o programas para el mejoramiento de los } \\
\text { márgenes de ingresos desde el cultivo }\end{array}$ \\
\hline
\end{tabular}

Fuente: Elaboración de los Autores

Es importante anotar que las posiciones frente a estos objetivos muestran una alta convergencia entre los actores, es decir, que la gran mayoría de los actores tuvieron posiciones favorables frente a los objetivos planteados, al ser consultados en el juego de actores (método MACTOR), sin embargo, al evaluar el grado de compromiso o importancia para el actor de os objetivos, se evidenciaron pocas convergencias fuertes que puedan dinamizar el proceso de construcción del futuro.

\section{Gráfico 4}

Convergencias y Divergencias Valoradas entre Actores
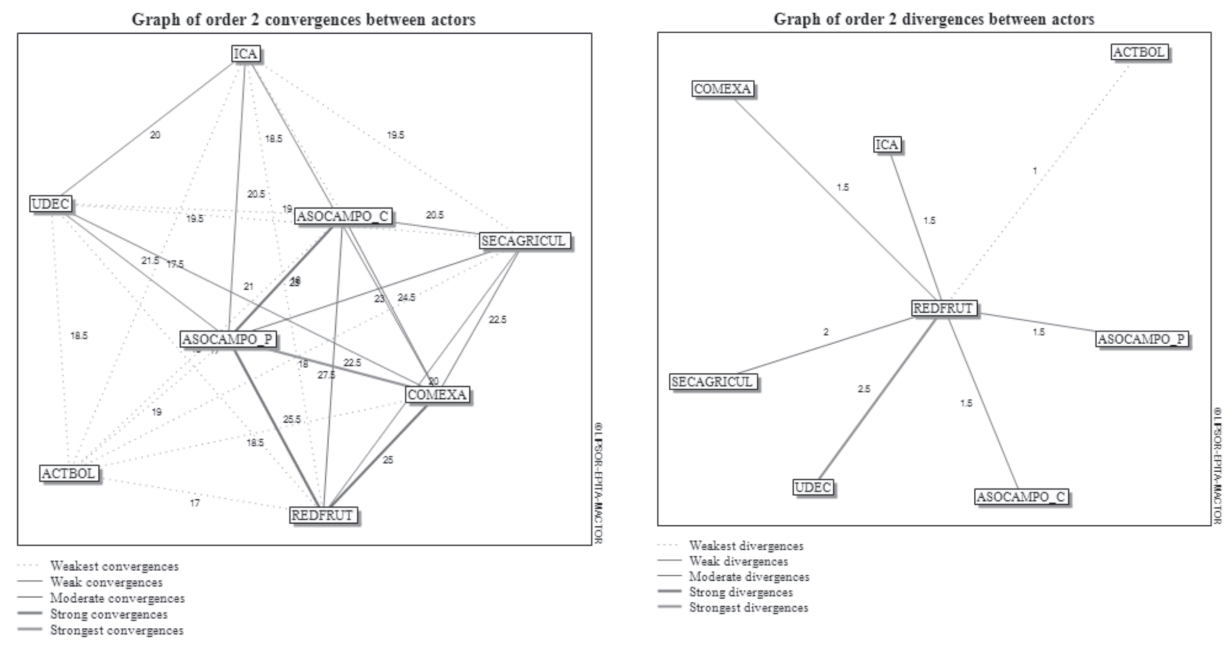

Fuente: Resultados Taller de Juego de Actores - Software MACTOR 
A partir de las convergencias cabe resaltar el alto y moderado nivel de convergencia entre actores como COMEXA, Asocampo productores (ASOCAMPO_P), Asocampo comercializadores (ASOCAMPO_C) y la Red frutícola de Bolívar (REDFRUT); además, que la convergencia entre el ICA y la Secretaría de Agricultura (SECAGRICUL) es de las más bajas, al igual que la convergencia de la U de C con esta última. Esto deja de manifiesto nuevamente una desarticulación, que es este caso refleja la poca asociatividad regional de los sectores de la academia e investigación respecto al gobierno y el sector productivo para adelantar proyectos productivos y de desarrollo empresarial, lo cual puede convertirse en una limitante para el desarrollo industrial de la cadena hortofrutícola de Bolívar, dadas las necesidades de incorporación de mejoras tecnológicas e innovaciones para mejorar la competitividad del sector.

Respecto a las divergencias mostradas por parte de la asociación Red frutícola en torno a la aplicación de herramientas biotecnológicas, es igualmente un factor a tener en cuenta dentro de los planes de expansión de la oferta frutícola del departamento, dado que estas resistencias pueden entrar a frenar proyectos o programas de mejoramiento productivo en los productos manejados por esta asociación.

\section{Conclusiones}

Los resultados del proyecto de investigación dejan ver la gran necesidad de intervenir el desarrollo industrial de las minicadenas hortifrutícolas del departamento de Bolívar, con el fin de hacerlas económicamente competitivas y socialmente sostenibles. Para ello se deja ver la clara necesidad de intervenir factores como: productos innovadores, biotecnología, Impacto ambiental, tecnologías de procesamiento, alianzas estratégicas, disposición a la asociatividad, acuerdos comerciales internacionales, productividad, vocación exportadora, márgenes de ingreso, entre otros. En estos factores se deja ver igualmente la gran tarea del sector para trabajar en la construcción de un futuro deseado dado que ello implica una gran modernización del sector y sobre todo un proceso de concertación y negociación sobre los objetivos planteados. Así también, se deja ver como se hace necesaria una mirada más atenta desde los medios académicos y científicos sobre la situación de este sector, en el sentido de desarrollar más soluciones que permitan articular mejor los procesos de producción y el desarrollo de mejores productos, así como una mejor atención de las instituciones para la colaboración y estado desde el ámbito territorial (departamento y municipios) para promover el desarrollo del sector. Pero sobre todo, la voluntad de los actores de la cadena productiva (productores- 
transformadores-comercializadores) en torno al desarrollo de un proyecto conjunto de largo plazo alrededor de los objetivos planteados en el presente documento, es un factor clave para el éxito, ya que estos son la razón de ser del cluster y su desarrollo.

\section{BIBLIOGRAFÍA}

CÁMARA DE COMERCIO DE BOGOTÁ. (2005) Balance tecnológico cadena productiva hortofrutícola en Bogotá y Cundinamarca. Bogotá.

DNP. Análisis de cadenas productivas. Estructura, comercio internacional y protección. Departamento Nacional de Planeación. Edición electrónica en: http://www.dnp.gov.co/ paginas_detalle.aspx?idp=336.

GODET, Michel. (2000) La caja de herramientas de la prospectiva estratégica. Cuadernos de LIPS No. 5. Laboratoire d'Investigation Prospective et Stratégique, Laboratoire d'Investigation Prospective et Stratégique.

GOMES, Antonio; Valle, Suzana; Pedroso, Carlos. (2002) Cadena productiva: Marco conceptual para apoyar la prospección tecnológica. Revista Espacios. Vol. 23 (2).

MOJICA, Francisco. (1992) La prospectiva. Técnicas para visualizar el futuro. Legis. Bogotá.

RAMOS, Joseph. (2001) Complejos productivos en torno a los recursos naturales: ¿una estrategia prometedora? Artículo publicado en: Martine, Dirven (compiladora). Apertura económica y (des)encadenamientos productivos. Reflexiones sobre el complejo lácteo en América Latina. CEPAL.

BIOGRAFIA

\section{JULIO AMÉZQUITA LÓPEZ}

Ingeniero Industrial, candidato a Magíster en Administración. Director del grupo de investigaciones CTS+I Unicartagena. Docente de tiempo completo en la Universidad de Cartagena - Facultad de Ciencias Económicas.

\section{ERIK BALDOVINO VILLACOB}

Economista. Investigador de la Cámara de Comercio de Cartagena. Miembro del grupo de investigaciones CTS+I Unicartagena. 